\title{
Towards a Circular Higgs and Electroweak Factory
}

\author{
Frank Zimmermann ${ }^{* \dagger}$ \\ CERN, 1211 Geneva 23, Switzerland \\ E-mail: frank.zimmermannecern.ch
}

\begin{abstract}
A large circular $\mathrm{e}^{+} \mathrm{e}^{-}$collider followed by a highest-energy hadron collider in the same tunnel promises the most far-reaching physics program for the post-LHC era, and such a facility could well serve the particle physics community through the end of the 21 st century. Two such projects are presently proposed: The Future Circular Collider, in a global collaboration hosted by CERN, and the combination of Circular Electron Positron Collider and Super Proton-Proton Collider, advanced by IHEP Beijing. Over the centre-of-mass energy range from 90 to about $365 \mathrm{GeV}$, covering all known heavy elements of the Standard Model, from the $\mathrm{Z}$ resonance to the top-quark threshold, the circular $\mathrm{e}^{+} \mathrm{e}^{-}$collider offers a high luminosity and exquisite energy efficiency. The high luminosity is maintained by top-up injection from a full-energy booster synchrotron. On the $\mathrm{Z}$ pole and at the WW threshold resonant depolarisation will allow for a precision energy calibration at the ppm level. This article summarizes and compares the designs of FCC-ee and CEPC, covering the latest accelerator layouts and beam parameters, R\&D plans, ongoing prototyping of key technologies, such as for the SRF system, and possible implementation schedules.
\end{abstract}

The European Physical Society Conference on High Energy Physics (EPS-HEP2021) 26-30 July 2021

Online conference, jointly organized by Universität Hamburg and the research center DESY

\footnotetext{
*Speaker.

†frank.zimmermann@ @ern.ch
} 


\section{Motivation and Goals}

Two similar, future large high-energy physics research infrastructures are being proposed: The Future Circular Collider (FCC), located in the Lake Geneva basin and linked to the existing CERN facilities, and the Circular Electron Positron Collider (CEPC) combined with the Super Proton-Proton Collider (SPPC), in China. Both the FCC and CEPC/SPPC integrated programs are inspired by the successful past Large Electron Positron collider (LEP) and Large Hadron Collider (LHC) projects at CERN. They offer a comprehensive long-term program, maximizing physics opportunities, with an $\mathrm{e}^{+} \mathrm{e}^{-}$collider as a possible first stage (FCC-ee or CEPC, respectively) serving as Higgs factory, electroweak and top factory at highest luminosities, and running at four different centre-of-mass energies, namely on the $\mathrm{Z}$ pole, at the $\mathrm{WW}$ threshold, at $\mathrm{ZH}$ production peak, and at the $t \bar{t}$ threshold. In a second stage, FCC-ee or CEPC would be followed by a highest-energy proton collider, either FCC-hh (with $100 \mathrm{TeV}$ centre-of-mass energy) or SPPC (at about $75 \mathrm{TeV}$ ), naturally succeeding the LHC at the energy frontier. The hadron colliders can also accommodate ion and lepton-hadron collision options, providing for complementary physics. The lepton and hadron colliders would profit from a common civil engineering and from also sharing the technical infrastructures. The FCC would also build on and reuse CERN's existing infrastructure, while CEPC/SPPC would be a green-field construction. The FCC and CEPC/FCC integrated projects would allow for a seamless continuation of High Energy Physics (HEP) after the completion of the High Luminosity LHC (HL-LHC) physics programme in the late 2030s.

\section{Lepton Collider Design}

Both FCC-ee [1] and CEPC [2] are conceived as double-ring colliders with a full-energy topup booster ring. CEPC evolved from an almost single ("partial double") ring design, with a size of $54 \mathrm{~km}$ like the earlier DLEP [3], to a true double ring of $\sim 100 \mathrm{~km}$ circumference, similar to FCC. The colliders feature two Interaction Points (IPs) each. Two other straights house the radiofrequency (RF) cavities. The strength of the arc magnets is tapered so as to match the local energy. An asymmetric interaction region (IR) layout limits the photon energies of the synchrotron radiation (SR) emitted by the incoming beams towards detectors and, at the same time, generates the large crossing angle [4]. A common use of the RF systems for both beams is planned at the highest-energy working point ( $\mathrm{t} \overline{\mathrm{t}})$, where each beam comprises only a few tens of bunches, to minimise the cost of construction. Key parameters for both colliders are compiled in Table 1.

Two important operational ingredients are "bootstrapping injection" and top-up injection [5]. Alternating re-injection to "top up" the circulating electron and positron bunches maintains approximately constant beam current and luminosity, so that the average luminosity of FCC-ee or CEPC approaches the peak luminosity. Intensities of colliding bunches must be kept equal to within a few percent to avoid a beam-beam flip-flop effect, where one bunch would blow up and the opposite bunch shrink, resulting in an unrecoverable situation. For the same reason, when filling the machine from zero, a bootstrapping injection process is foreseen, with alternating injections into either of the two collider rings, avoiding large charge imbalances [5].

At the FCC-ee, highly precise energy calibration using resonant depolarisation at c.m. energies of 91 and $160 \mathrm{GeV}$, with wiggler-polarised pilot bunches, and roughly $10^{5}$ times higher luminosity 
Table 1: Key parameters of FCC-ee (CDR 2018 [1]) and CEPC (TDR 2021 [2]). Luminosity values are given per interaction point (IP). Both the natural bunch lengths due to synchrotron radiation (SR) and their collision values including beamstrahlung (BS) are shown. CEPC considers a single-frequency $(650 \mathrm{MHz})$ RF system, the FCC-ee CDR a combination of $400 \mathrm{MHz}$ (at the first three energies, up to $2 \times 2 \mathrm{GV}$ ) and 800 $\mathrm{MHz}$ (additional cavities for ttbar operation, providing $6.9 \mathrm{GV}$ ), with respective voltage strengths indicated.

\begin{tabular}{|l|cccc|cccc|}
\hline \hline & \multicolumn{4}{|c|}{ FCC-ee } & \multicolumn{4}{c|}{ CEPC } \\
\hline Beam energy (GeV) & 45.6 & 80 & 120 & 182.5 & 45.5 & 80 & 120 & 180 \\
Bunches/beam & 16640 & 2000 & 328 & 48 & 11951 & 1297 & 249 & 35 \\
Beam current [mA] & 1393 & 147 & 29 & 5.4 & 803 & 84 & 17 & 3.3 \\
Luminosity/IP [10 ${ }^{34} \mathrm{~cm}^{-2} \mathrm{~s}^{-1}$ ] & 230 & 28 & 8.5 & 1.6 & 115 & 16 & 5.0 & 0.5 \\
Energy loss / turn [GeV] & 0.036 & 0.34 & 1.72 & 9.2 & 0.037 & 0.36 & 1.8 & 9.1 \\
Synchr. rad. power [MW] & 100 & 100 & 100 & 100 & 60 & 60 & 60 & 60 \\
RF voltage [GV] & 0.10 & 0.75 & 2.0 & $4.0+6.9$ & 0.12 & 0.70 & 2.2 & 10 \\
Rms bunch length (SR) [mm] & 3.5 & 3.0 & 3.2 & 2.0 & 2.5 & 2.5 & 2.3 & 2.2 \\
Rms bunch length (+BS) [mm] & 12 & 6.0 & 5.3 & 2.5 & 8.7 & 4.9 & 3.9 & 2.9 \\
Rms hor. emittance $\varepsilon_{x, y}[\mathrm{~nm}]$ & 0.27 & 0.84 & 0.63 & 1.5 & 0.27 & 0.87 & 0.64 & 1.4 \\
Rms vert. emittance $\varepsilon_{x, y}[\mathrm{pm}]$ & 1.0 & 1.7 & 1.3 & 2.9 & 1.4 & 1.7 & 1.3 & 4.7 \\
Longit. damping time [turns] & 1273 & 236 & 70 & 20 & 1230 & 224 & 67 & 20 \\
Vertical IP beta $\beta_{y}^{*}[\mathrm{~mm}]$ & 0.8 & 1.0 & 1.0 & 1.6 & 0.9 & 1.0 & 1.0 & 1.7 \\
Beam lifetime [min.] & 68 & 59 & 12 & 12 & 80 & 55 & 12 & 18 \\
\hline \hline
\end{tabular}

than LEP, will allow measuring the masses of the $\mathrm{Z}$ and $\mathrm{W}$ bosons, as well as the width of the $\mathrm{Z}$, with 20-50× improved precision, rendering the FCC-ee an exceptional electroweak factory [6]. Similar precision should be attainable at CEPC.

In addition to the 4 baseline running modes shown in Table 1, another optional operation mode is presently under investigation for FCC-ee, namely the direct $s$-channel Higgs production, $\mathrm{e}^{+} \mathrm{e}^{-} \rightarrow \mathrm{H}$, at a centre-of-mass energy of $125 \mathrm{GeV}$. Here, a monochromatization scheme should reduce the effective collision energy spread so as to become comparable to the width of the Higgs [7]. This type of operation would provide experimental access to the electron Yukawa coupling.

\section{R\&D}

The superconducting RF system is the underpinning technology for the lepton colliders. The FCC-ee baseline foresees the use of single-cell $400 \mathrm{MHz} \mathrm{Nb/Cu}$ cavities at the $\mathrm{Z}$, four-cell 400 $\mathrm{MHz} \mathrm{Nb/Cu}$ cavities at the $\mathrm{W}$ and $\mathrm{H}(\mathrm{ZH})$ energies, and a complement of five-cell bulk $\mathrm{Nb} 800$ $\mathrm{MHz}$ cavities at $2 \mathrm{~K}$ for $\mathrm{t} \overline{\mathrm{t}}$ operation [1]. In the booster, only multi-cell 400 and $800 \mathrm{MHz}$ cavities will be installed. For the collider, also alternatives, with possibly fewer changes between operating points, are being explored, such as novel $600 \mathrm{MHz}$ slotted waveguide elliptical (SWELL) cavities [8]. CEPC plans to install two-cell bulk $\mathrm{Nb} 650 \mathrm{MHz}$ elliptical cavities at $2 \mathrm{~K}$ in its collider rings, 
and 1.3 GHz ILC type cavities in the booster [2]. A new CEPC SRF technology platform has started operation in Beijing (Huairou area), which can produce and deliver about SRF 20 cryomodules per year. Advanced high-efficiency klystrons are being prototyped and tested for either collider.

For the FCC-ee it is planned to build a complete arc half-cell mock up including girder, vacuum system with antechamber and pumps, dipole, quadrupole and sextupole magnets, beam-position monitors, cooling and alignment systems, and technical infrastructure interfaces, by 2025, along with prototypes of key beam diagnostics, like bunch-by-bunch longitudinal charge-density monitors, ultra-low emittance measurements, beam-loss and beamstrahlung monitors, polarimeter and luminometer. A novel positron source plus capture linac, and the achievable positron yield, will be tested with a primary electron beam of variable energy $(0.4-6 \mathrm{GeV})$ at the PSI SwissFEL.

The injection energy for the booster is defined by the field quality of its low-field magnets. For the CEPC booster, short models of dipole magnets with either diluted iron core or without iron core (cosine theta) were designed, built and tested. For an injection energy above $20 \mathrm{GeV}$, the iron core magnets could be used. Lower energy might be achieved with the iron-less dipole magnets, of which a full-scale $5.1 \mathrm{~m}$ long prototype is under construction.

The SuperKEKB collider, presently being commissioned [9], features many of the key elements of FCC-ee or CEPC: double ring, large crossing angle, low vertical IP beta function $\beta_{y}^{*}$ (design value $\sim 0.3 \mathrm{~mm}$ ), short design beam lifetime of a few minutes, top-up injection, and $\mathrm{e}^{+}$ rate of up to several $10^{12} / \mathrm{s}$. SuperKEKB has already achieved, in both rings, the world's smallest ever $\beta_{y}^{*}$ of $0.8 \mathrm{~mm}$, which is the smallest value being considered for FCC-ee. Profiting from a new "virtual" crab-waist collision scheme, first developed for the FCC-ee [4], in June 2021 SuperKEKB reached a world record luminosity of $3.12 \times 10^{34} \mathrm{~cm}^{-2} \mathrm{~s}^{-1}$ [9]. However, many issues still need to be addressed, such as a vertical emittance larger than expected even at low intensity or without collision, collimator impedance and single-bunch instability threshold, unexplained sudden beam loss without any beam oscillation, insufficient quality of the injected beam, etc. Other important beam studies relevant for FCC-ee and CEPC are being conducted at INFN-LNF/DAFNE, DESY/PETRA III, BINP/VEPP-4M, and KIT/KARA [10].

\section{Hadron Colliders}

FCC-ee and CEPC would each be followed by a subsequent hadron collider, called FCC-hh or SPPS, respectively. Table 2 compares selected parameters of these two machines with the design values for the LHC and its high-luminosity upgrade HL-LHC. The proposed two future hadron colliders aim for about an order of magnitude performance increase in both energy and luminosity compared with the LHC. The $100 \mathrm{TeV}$ c.m. collision energy of FCC-hh should be compared with the $14 \mathrm{TeV}$ design energy of the LHC. FCC-hh is expected to deliver $20 \mathrm{ab}^{-1}$ for each of two experiments collected over 25 years of operation, and CEPC about $10 \mathrm{ab}^{-1}$ over the same period of time, while the target value for the full LHC/HL-LHC programme is $3 \mathrm{ab}^{-1}$. The FCC-hh represents a similar performance increase from LHC/HL-LLHC as the step from the Tevatron to the LHC. The key technology on which the hadron colliders are based is high-field magnets. FCChh considers the use of $\mathrm{Nb}_{3} \mathrm{Sn}$ superconductor with a dipole field of $16 \mathrm{~T}$, SPPS an iron-based high-temperature superconductor with $12 \mathrm{~T}$ field. In addition to the magnets, SR poses another challenge for future hadron colliders, as SR power at MW levels is emitted inside the cold magnets 
Table 2: Key parameters of FCC-hh (CDR 2018 [11]) and SPPC (CDR 2018 [12]).

\begin{tabular}{|c|c|c|c|c|}
\hline & FCC-hh & SPPC & HL-LHC & "LHC \\
\hline Centre-of-mass energy $(\mathrm{TeV})$ & 100 & 75 & 14 & 14 \\
\hline Dipole field [T] & 16 & 12 & 8.33 & 8.33 \\
\hline Circumference $[\mathrm{km}]$ & 97.756 & 100 & 26.7 & 26.7 \\
\hline Beam current $[\mathrm{A}]$ & 0.5 & 0.73 & 1.1 & 0.58 \\
\hline Bunch intensity $\left[10^{11}\right]$ & 1 & 1.5 & 2.2 & 1.15 \\
\hline Bunch spacing [ns] & $25 \quad 25$ & 25 & 25 & 25 \\
\hline Synchr. radiation power $[\mathrm{kW}]$ & 4800 & 2400 & 15 & 7 \\
\hline SR power / length $[\mathrm{W} / \mathrm{m} /$ aperture $]$ & 28.4 & 14 & 0.33 & 0.17 \\
\hline Longit. emit. damping time $[\mathrm{h}]$ & 0.54 & 1.2 & 12.9 & 12.9 \\
\hline IP beta function $\beta_{x, y}^{*}[\mathrm{~m}]$ & $1.1 \quad 0.3$ & 0.75 & 0.15 (min.) & 0.55 \\
\hline Normalized rms emittance $[\mu \mathrm{m}]$ & 2.1 & 2.4 & 2.5 & 3.75 \\
\hline Peak luminosity $\left[10^{34} \mathrm{~cm}^{-2} \mathrm{~s}^{-1}\right]$ & 30 & 10 & 5 (lev.) & 1 \\
\hline Events / bunch crossing & $170 \quad 1000$ & $\sim 300$ & 132 & 27 \\
\hline Stored energy beam [GJ] & 8.4 & 9.1 & 0.7 & 0.36 \\
\hline
\end{tabular}

of the collider arcs. Therefore, the cryo-beam vacuum system and the effective SR heat removal call for innovative, energy-efficient solutions.

\section{Possible Implementation Schedules}

The technical schedule of the FCC integrated project foresees the start of FCC tunnel construction around the year 2030 - or three years after a project approval - , the first $\mathrm{e}^{+} \mathrm{e}^{-}$collisions at the FCC-ee in 2040 or during the early 2040s, and the first FCC-hh hadron collisions by 2065 see Fig. 1. For CEPC, the start of tunnel construction is expected five years earlier, in 2025, and $\mathrm{e}^{+} \mathrm{e}^{-}$data taking in $\sim 2033$, that is seven years before FCC-ee. However, the beginning of SPPC hadron collisions is roughly forecast for the year $\sim 2065$, the same target date as for FCC-hh.

\section{Conclusions}

Two circular Higgs and electroweak factories are being developed, FCC-ee and CEPC. Both promise highly efficient performance over a large energy range, extending from $\mathrm{Z}$ pole to the $\overline{\mathrm{t}}$ threshold. Independent design efforts in Europe and in China have led to remarkably similar layouts and parameters. Further improvements or additions appear nevertheless possible, e.g. monochromatised s-channel H production, operation with 4 IPs instead of 2, etc. Synergetic R\&D efforts with prototyping of key components are underway. Either machine would prepare for a subsequent hadron collider, FCC-hh or SPPC, support additional collider options, and enable a frontier-physics programme spanning over a century. FCC has now entered the Feasibility Study phase, results of which will inform the next update of European Strategy for Particle Physics expected in 2026/27. 


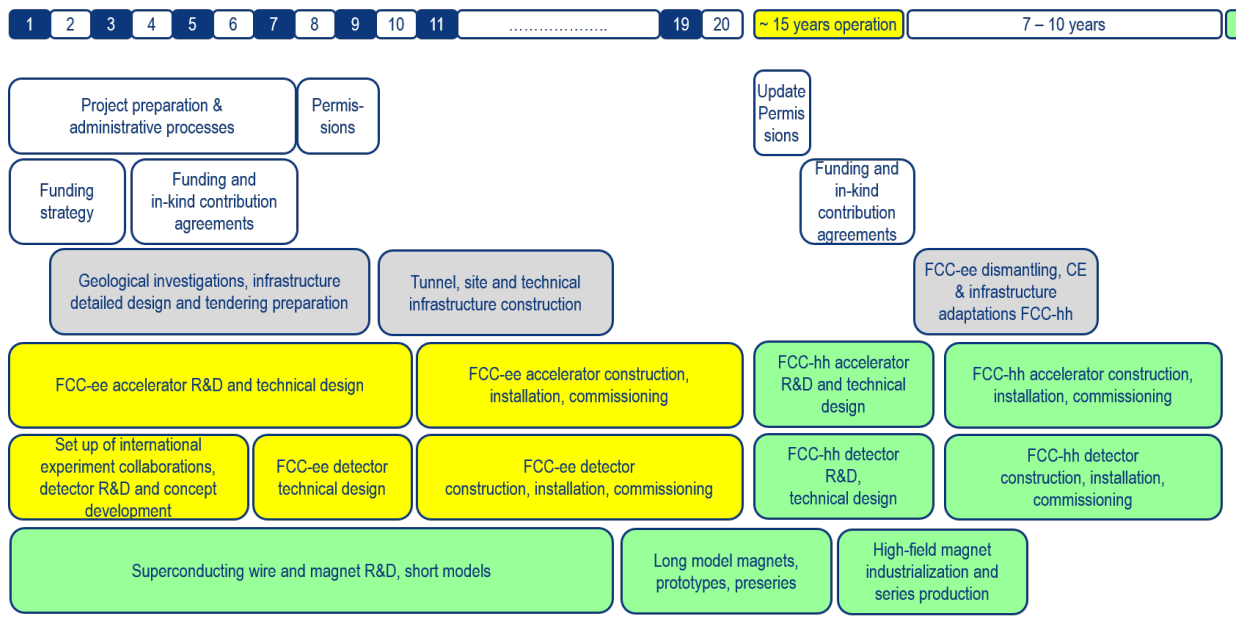

Figure 1: Technical schedule of the FCC integrated project with year 1 equal to 2021 (M. Benedikt).

\section{Acknowledgements}

I am indebted to the FCC Collaboration and to the CEPC Design Team. I thank M. Benedikt and J. Gao, for providing key material on FCC-ee and CEPC, respectively, and B. Dalena, J. Osterhoff, and R. Tomás, for encouraging my presentation. This work was supported by the European Union's Horizon 2020 Research and Innovation programme under grant no. 951754 (FCCIS).

\section{References}

[1] M. Benedikt et al., "Future Circular Collider, Vol. 2, The Lepton Collider (FCC-ee)", EPJ ST 228, 2 (2019), 261-623.

[2] J. Gao, private communication, 5 July 2021.

[3] A. Blondel and F. Zimmermann, "A High Luminosity e+e- Collider in the LHC tunnel to study the Higgs Boson”, arXiv 1112.2518 (2012).

[4] K. Oide et al., Phys. Rev. Accel. Beams 19, 11, 111005 (2016).

[5] D. Shatilov, "FCC-ee Parameter Optimization”, ICFA Beam Dyn. Newsl. 72 (2017) 30-41.

[6] A. Blondel et al., "Polarization and ... Energy Calibration at FCC-ee", arXiv 1909.12245 (2019).

[7] A. Faus-Golfe, M.A. Valdivia Garcia, F. Zimmermann, "The Challenge of Monochromatization Direct s-Channel Higgs Production: $\mathrm{e}^{+} \mathrm{e}^{-} \rightarrow \mathrm{H}$ ", submitted for publication (2020).

[8] I. Syratchev, F. Peauger, I. Karpov, O. Brunner "A Superconducting Slotted Waveguide Elliptical Cavity for FCC-ee (1.0)," Zenodo; https://doi.org/10.5281/zenodo.5031953 (2021).

[9] Y. Ohnishi, "Status and perspectives of the SuperKEKB project" these proceedings (2021).

[10] J. Keintzel et al., "Experimental beam tests for FCC-ee," these proceedings (2021).

[11] M. Benedikt et al., "Future Circular Collider, Vol. 3, The Hadron Collider (FCC-hh)", EPJ ST 228, 2 (2019), 755-1107.

[12] The CEPC Study Group, “CEPC Conceptual Design Report: Volume 1 - Accelerator”, arXiv 1809.00285 (2018). 